\title{
Laboratory simulations of astrophysical jets and solar coronal loops: new results
}

\author{
P. M. Bellan, D. Kumar, E. V. Stenson, S. K. P. Tripathi, G. S. Yun, and A. L. Moser \\ Applied Physics, Caltech, Pasadena, CA 91125, USA
}

\begin{abstract}
An experimental program underway at Caltech has produced plasmas where the shape is neither fixed by the vacuum chamber nor fixed by an external coil set, but instead is determined by self-organization. The plasma dynamics is highly reproducible and so can be studied in considerable detail even though the morphology of the plasma is both complex and time-dependent. A surprising result has been the observation that self-collimating MHD-driven plasma jets are ubiquitous and play a fundamental role in the self-organization. The jets can be considered lab-scale simulations of astrophysical jets and in addition are intimately related to solar coronal loops. The jets are driven by the combination of the axial component of the $\mathbf{J} \times \mathbf{B}$ force and the axial pressure gradient resulting from the non-uniform pinch force associated with the flared axial current density. Behavior is consistent with a model showing that collimation results from axial non-uniformity of the jet velocity. In particular, flow stagnation in the jet frame compresses frozen-in azimuthal magnetic flux, squeezes together toroidal magnetic field lines, thereby amplifying the embedded toroidal magnetic field, enhancing the pinch force, and hence causing collimation of the jet.
\end{abstract}

\section{INTRODUCTION}

The magnetohydrodynamic (MHD) equations have no intrinsic scale so in principle MHD phenomena occurring in space and astrophysical situations should be reproducible in laboratory experiments providing the appropriate boundary conditions are imposed. Two types of boundary conditions are being implemented in experiments at Caltech in order to investigate MHD plasma configurations relevant to space and astrophysical situations. The experiments are not expected or intended to be exact scale models, because it is in general not possible to duplicate the extreme parameters of space and astrophysical phenomena. Nevertheless, the experiments provide useful qualitative insights into certain space and astrophysical situations because the experiments are arranged to be governed by the same dynamical relationships and to have similar morphology.

One experiment is designed to be relevant to astrophysical jets and the other to solar coronal loops; the experimental layouts and images of typical plasmas are shown in Figs. 1 and 2. Both the astrophysical jet $[1,2,3,4,5]$ and solar coronal loop experiments $[6,7,8]$ use technology derived from spheromak devices developed previously for magnetic fusion applications [9]. The astrophysical jet experiment is axisymmetric so that its magnetic fields can be decomposed into toroidal and poloidal components whereas the solar coronal loop experiment has bipolar symmetry analogous to a horseshoe magnet.

Both experiments are governed by the interplay between three basic equations of MHD, namely the induction equation

$$
\frac{\partial \mathbf{B}}{\partial t}=\nabla \times(\mathbf{U} \times \mathbf{B})
$$

the equation of motion

$$
\rho \frac{d \mathbf{U}}{d t}=\mathbf{J} \times \mathbf{B}-\nabla P
$$

and the continuity equation

$$
\frac{\partial \rho}{\partial t}+\nabla \cdot(\rho \mathbf{U})=0
$$

The induction equation constrains the magnetic flux to be frozen into the frame of the plasma while the equation of motion states that the plasma is accelerated by the sum of magnetic and hydrodynamic forces. Typical plasma 


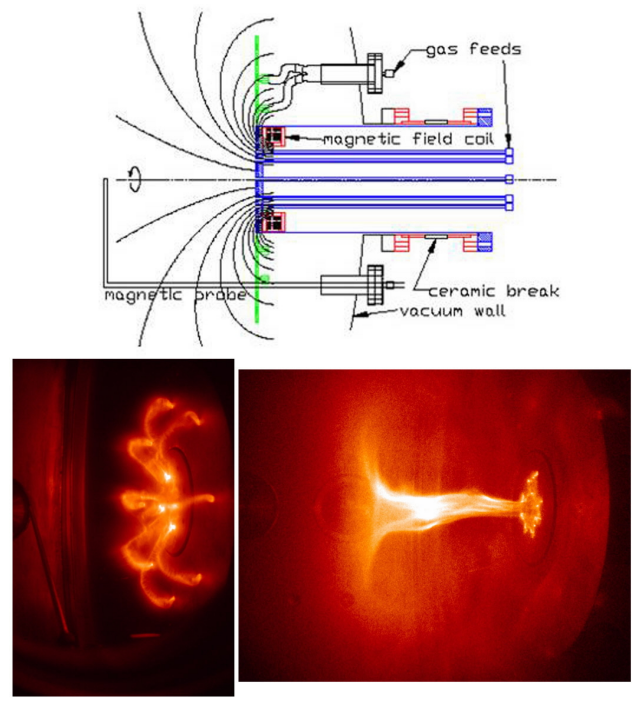

FIGURE 1. Top: Layout of plasma gun used for astrophysical jet experiment. Magnetic field coil establishes poloidal bias field and then gas valves inject neutral gas from orifices in electrodes. High voltage applied between inner and outer electrodes breaks down gas to form spider legs shown in lower left. Inner segments of spider legs merge to form jet shown in lower right.

densities are $10^{21}-10^{23} \mathrm{~m}^{-3}$, typical temperatures are a few eV, typical magnetic fields are $10^{-2}-10^{-1} \mathrm{~T}$, and the characteristic scale length $L$ is $2-50 \mathrm{~cm}$.

The experiment takes place in a vacuum chamber much larger than the plasma configuration so that the plasma morphology can evolve as prescribed by the MHD equation of motion without being affected by interaction with walls. In order for MHD dynamics to be manifested, the experimental time duration is arranged to be longer than the characteristic time scale of MHD dynamics, namely the Alfvén time scale $\tau_{A}=L / v_{A}$ where $v_{A}$ is the Alfvén velocity. In addition, the duration of the experiment is arranged to be shorter than the resistive diffusion time $\tau_{R}=\mu_{0} L^{2} / \eta$ where $\eta$ is the resistivity so that magnetic flux is frozen into the plasma, i.e., Eq.1 is satisfied. Thus, the characteristic time $\tau$ of the experiment must be such that $\tau_{A} \ll \tau \ll \tau_{R}$. For this to be possible it is necessary for the Lundquist number $S=\tau_{R} / \tau_{A}$ to be much larger than unity. The experimental Lundquist number is $S \sim 10^{2}-10^{3}$ and the condition $\tau_{A} \ll \tau \ll \tau_{R}$ is satisfied by having the experimental duration be of the order of $10 \mu \mathrm{s}$ with significant dynamical change occurring on a time scale of $1 \mu \mathrm{s}$. This fast time scale has the virtue that the required $\sim 10^{8} \mathrm{~W}$ pulsed power can be achieved using modest-size capacitor banks and furthermore the short duration of the pulse is such that there is no significant heating of the electrodes or of probes inserted into the plasma.

The typical sequence of operation for both experiments is as follows:

(i) A bias magnetic field produced by electric current in an external coil is established on a $\sim 5 \mathrm{~ms}$ time scale; this long time scale compared to the time scale of subsequent plasma dynamics allows the bias magnetic flux to diffuse into the electrodes before the plasma dynamics occurs and then remain "frozen-in" to the electrodes on the time scale of the plasma dynamics. The bias magnetic field in the astrophysical jet experiment is provided by a single coil as shown in Fig. 1, while the bias magnetic field in the solar coronal loop experiment is produced by two coils which together make an arched magnetic field as shown in Fig. 2. In both cases the bias magnetic field links the two electrodes.

(ii) Fast gas valves are used to puff a cloud of gas from orifices in the electrodes.

(iii) High voltage, typically $3-6 \mathrm{kV}$, is applied from a capacitor bank across the electrodes; this breaks down the gas to form an initial low-density plasma.

(iv) The discharging capacitor bank ramps up a current in $\sim 5-10 \mu \mathrm{s}$ having maximum value of 50-80 kA for the solar experiment and 100-150 kA for the astrophysical jet experiment. This current flows from the anode electrode to 

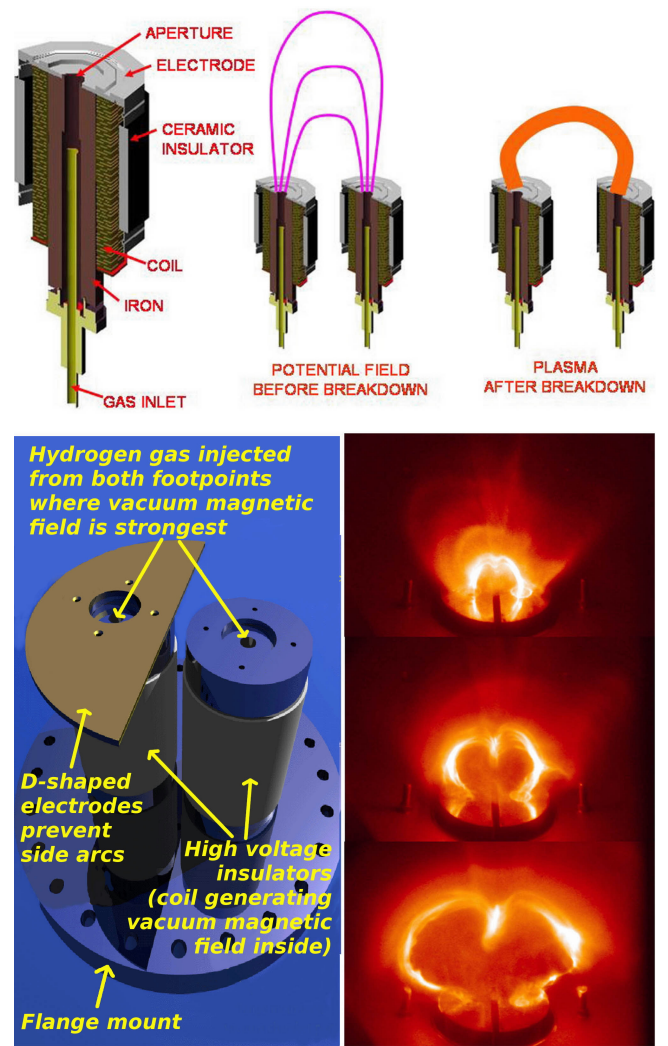

FIGURE 2. Top left shows electromagnet with electrode and gas feed used for solar coronal loop simulation. Top middle sketches vacuum magnetic field produced by pair of opposite polarity electromagnets. Top right sketches plasma after breakdown. Bottom left shows actual pair of electromagnets, electrode (right electrode not shown for clarity), and gas feeds. Bottom right shows images of typical sequence.

the cathode electrode approximately following the bias magnetic field linking the electrodes.

(v) Magnetic forces resulting from the interaction between this current and its self-generated magnetic field pump additional plasma from the orifices into the flux tube linking the electrodes and also cause the flux tube to lengthen and deform. The plasma with its frozen-in combination of bias and self-generated magnetic fields evolves through a complex sequence of reproducible morphologies.

(vi) The evolving plasma with frozen-in magnetic field is observed using a variety of diagnostics. The observations are compared with theoretical models and used to motivate new models.

\section{ASTROPHYSICAL JET EXPERIMENT}

The two electrodes in the astrophysical jet experiment are shown in Fig. 1 and consist of a $20 \mathrm{~cm}$ diameter copper disk coplanar and coaxial with a copper annulus having $50 \mathrm{~cm}$ outside diameter. A gap of a few mm separates the disk 


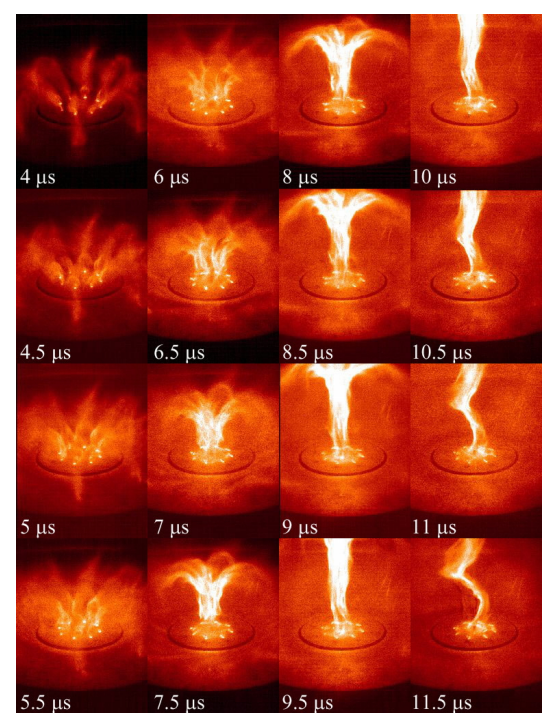

FIGURE 3. Typical sequence in astrophysical jet experiment (from Ref. [3]).

from the annulus so that the disk and annulus can be at different voltages. The disk and annulus each have eight gas injection orifices arranged in a circle. When the high-voltage capacitor bank is connected by an ignitron switch across the two electrodes, the injected gas breaks down to form eight plasma-filled arched flux tubes [2,3] as shown in the 4 $\mu$ s frame of Fig.3. Each arched plasma-filled flux tube spans a disk gas injection orifice and a corresponding annulus gas injection orifice. The eight flux tubes approximately follow the initial vacuum poloidal magnetic field produced by the coil resulting in a configuration reminiscent of the eight legs of a spider. Because the electric currents in the inner segments of the spider legs (i.e., near the symmetry axis) are nearly parallel and are adjacent to each other, these inner segments mutually attract and merge resulting in the formation of a central column as shown in the 4.5 to $6.5 \mu \mathrm{s}$ frames of Fig.3.

The central column then lengthens forming a plasma jet. The jet length increases and the jet remains highly collimated until, at a critical length, it undergoes a rapid and very distinct kink instability. The threshold for the kink is in good agreement $[2,10]$ with the Kruskal-Shafranov kink instability theory. Jet axial velocities are in the range of $10-50 \mathrm{~km} / \mathrm{s}$ as measured by high-speed imaging, time-of-flight interferometer measurements, and Doppler shifts. The time-of-flight measurements [5] involve determining the time at which the jet front traverses a helium-neon laser interferometer [11] beam oriented perpendicular to the jet path. This time is resolved with high precision because of the rapid change in the interferometer signal when the jet front traverses the laser beam.

As shown in Fig. 4 the jet velocity is determined to be linearly proportional to the electric current $I$ and inversely proportional to the square root of the gas mass with scaling

$$
U=\frac{\mu_{0} I}{2 \pi a \sqrt{n m_{i}}}
$$

where $a$ is the jet radius at the electrode and $n$ is the density at the axial position where $U$ is measured. The $m_{i}^{-1 / 2}$ ion mass dependence was independently verified by comparing the velocity measured in a hydrogen gas experiment to the velocity in a deuterium gas experiment with all other parameters kept the same. The nominal $50 \mathrm{~km} / \mathrm{s}$ jet velocity is much faster than thermal velocities of ions or neutral gas. The scaling given by Eq.4 is in good agreement with the interpretation that the jet flow results from a two-part process. The first part of this process is the essentially instantaneous development of radial pressure balance between the hydrodynamic pressure and the magnetic pinch 


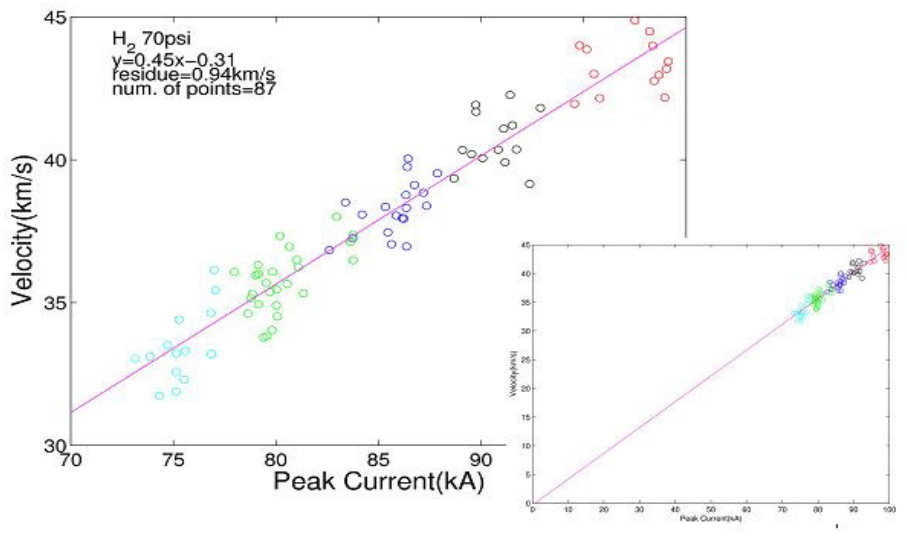

FIGURE 4. Jet velocity v. gun current meausured using time of flight. Inset at lower right is same data plotted with origin shown demonstrating that velocity is linearly proportional to current.

force so that the radial component of Eq. 2 gives

$$
P(0, z)=\frac{B_{\phi}^{2}(a, z)}{2 \mu_{0}}
$$

where for a given $z, P(0, z)$ is the pressure at $r=0$ and $B_{\phi}(a, z)$ is the azimuthal magnetic field at the radius $a$ of the current channel. The second part of the process involves a Bernoulli-type relation such that the axial component of Eq. 2 gives on the $z$ axis

$$
P+\frac{\rho U_{z}^{2}}{2}=\text { const }
$$

Combining Eqs.5 and 6 gives

$$
\frac{B_{\phi}^{2}(a, z)}{2 \mu_{0}}+\frac{\rho(0, z) U_{z}^{2}(0, z)}{2}=\text { const. }
$$

Near the electrodes $U_{z}(0, z)$ is small and $B_{\phi}(a, z)$ is large since the current channel radius $a$ is small. In contrast, far from the electrodes $U_{z}(0, z)$ is large and $B_{\phi}(a, z)$ is small because the current channel has flared out. Thus $\rho U_{z}^{2}$ evaluated at large $z$ and $r=0$ is approximately equal to $B_{\phi}^{2} / 2 \mu_{0}$ evaluated at small $z$ and $r=a$. Combining this result with Ampere's law $B_{\phi}=\mu_{0} I / 2 \pi a$ leads to Eq.4.

The observed collimation has motivated a model [12] that takes into account how compressibility in the axial direction affects the induction equation. According to this model, axial nonuniformity of the jet velocity whereby the jet tip moves slower than the immediately trailing jet segment results in a pile-up of plasma as the faster trailing segment catches up with the slower-moving tip. This pile-up results in an axial compression of plasma near the moving tip and hence an axial compression of the toroidal magnetic flux frozen into the plasma. This axial compression increases the density of the frozen-in toroidal flux and so increases the toroidal magnetic field. Because pinching is caused by the toroidal field, this enhances the magnetic pinch force so that the plasma radius decreases and the jet becomes collimated. 
This argument can be seen mathematically by expressing the toroidal component of Eq. 1 as

$$
\left(\frac{\partial}{\partial t}+\mathbf{U} \cdot \nabla\right) \frac{B_{\phi}}{r}=\mathbf{B} \cdot \nabla \frac{U_{\phi}}{r}-\frac{B_{\phi}}{r} \nabla \cdot \mathbf{U}
$$

and expressing the continuity equation as

$$
\left(\frac{\partial}{\partial t}+\mathbf{U} \cdot \nabla\right) \rho=-\rho \nabla \cdot \mathbf{U}
$$

so that elimination of $\nabla \cdot \mathbf{U}$ gives

$$
\frac{d}{d t}\left(\frac{B_{\phi}}{r}\right)=\frac{B_{\phi}}{\rho r} \frac{d \rho}{d t}
$$

where $d / d t=\partial / \partial t+\mathbf{U} \cdot \nabla$ is the time derivative seen by an observer in the plasma frame. Thus, axial compression of the plasma due to fast plasma catching up with slow plasma leads to an increase in $\rho$ and a proportional increase in $B_{\phi}$. This process corresponds to $\nabla \cdot \mathbf{U}$ being negative, i.e., to a converging flow.

A twelve-channel gated spectrometer system provides time- and space-resolved measurements of plasma density using the Stark effect and of velocity using Doppler shifts [4, 13]. The time- and space-resolved velocity and density measurements show that the jet tip indeed slows down and that there is a pile-up of plasma as faster-moving trailing plasma collides with the slower plasma at the jet tip [4, 13]; i.e., $\nabla \cdot \mathbf{U}$ is negative.

\section{Gas target experiment}

A target cloud of initially neutral gas has been placed in the path of the laboratory astrophysical jet. The target cloud is puffed using a fast gas valve. By varying the valve timing, the location and size of the target cloud can be controlled. Different combinations of jet gas and target gas have been used, the important distinction being the relative mass of jet and target gas species. It is observed that when a light-mass jet impacts a heavy-mass target cloud, the jet is effectively stopped, but in the opposite situation the target gas cloud bounces off from the jet like a light object at rest being impacted by a heavy object. In the light-jet/heavy-target situation, the jet is observed to pile-up when it impacts the gas cloud and the frozen-in magnetic flux also piles up resulting in an increase in magnetic flux density, i.e., an increase in magnetic field. An approximate doubling of the peak magnetic field frozen into the jet is observed in such a collision; this field amplification is qualitatively consistent with the predictions of Eq.10. The target gas is quickly ionized during the collision so the target cloud is effectively a plasma target.

\section{SOLAR CORONAL LOOP EXPERIMENT}

The solar coronal loop experiment has different geometry, there is still a jet flow, albeit more complex, and consideration of the interaction between this flow and the magnetic field contributed to the model [12] showing how plasma flow and collimation are mutually interconnected. This model is referred to as the "gobble" model because it predicts that the MHD force effectively ingests plasma from the electrode gas orifices and then accelerates this plasma to flow as a jet into the magnetic flux tube intercepting the electrode. The jet flows from small-radius regions of the flux tube to large-radius regions because, as discussed above in the context of the astrophysical jet experiment, the axial force is ultimately due to the axial gradient of $B_{\phi}^{2}$ where $\phi$ is the local azimuthal angle around the flux tube and $z$ is the distance along the axis of the flux tube. Because Ampere's law gives $B_{\phi}=\mu_{0} I / 2 \pi r$ where $r$ is the minor radius of the arched flux tube, $B_{\phi}$ is larger near the electrodes ( $r$ is small near the electrode) and small far from the electrode ( $r$ becomes larger on moving away from the electrode); this is indicated in the top middle sketch in Fig.2 where the flux tube field lines are closer together near the electrodes than at the apex of the arch.

In the solar experiment the arched magnetic field lines linking the two footpoint electrodes thus initially (i.e., when the field is vacuum-like) spread out at the apex of the arch and come together at the footpoints. Hence, when current starts to flow along these field lines from one electrode to the other, the local azimuthal magnetic field $B_{\phi}$ due to the current is smallest at the apex and largest at the footpoints. The gobble model thus predicts that there should be axial flows from both cathode and anode footpoints into the flux tube so that the flux tube should fill with plasma ingested 
from the two footpoints. To test this hypothesis, a series [14] of experiments was conducted in which a different gas was used at each of the two footpoints. In a typical experiment, hydrogen was injected from the cathode electrode and nitrogen from the anode electrode, or vice versa. The laboratory simulation of a coronal loop was then imaged with atomic line filters placed in front of the camera lens so as to image only the atomic line emission of a single species. For example, an $\mathrm{H}_{\alpha}$ filter was first used and then the experiment was repeated with a nitrogen ion atomic line filter. The images from the two identical plasma shots were then given false colors (e.g., red for hydrogen and green for nitrogen) and a composite figure was constructed showing both hydrogen and nitrogen. As shown in Fig. 5 the composite figure clearly shows a red jet (hydrogen) emanating from the cathode (top) and a green jet (nitrogen) emanating from the anode (bottom) with the two jets colliding very distinctly and together filling up the flux tube with plasma. The jets lengthen, but because the major radius of the arch increases in the same proportion, the system increases self-similarly and the jets never become interspersed. Because the light hydrogen jet is faster, the location of the jet-jet collision is not at the apex of the arch but instead is closer to the electrode from which the heavy nitrogen jet originates.
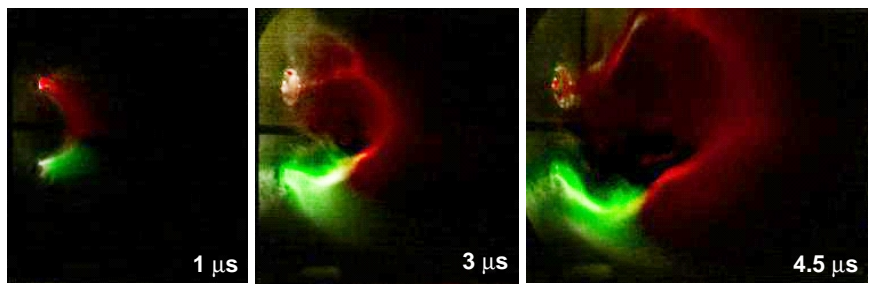

FIGURE 5. Composite image of hydrogen (from the cathode, red) and nitrogen (from the anode, green) jets coming from the top and bottom footpoints respectively and filling up arched flux tube spanning footpoints.

\section{Radially unstable motion of heavy ions}

In certain circumstances a relatively diffuse conical jet [8] was observed to emanate from the apex of the solar coronal loop experiment; an example is shown in Fig.6. This jet was phenomenologically different from the MHD jets described above but occurred in conjunction with a certain parameter regime of the MHD jets. To distinguish this new jet from the MHD jet, the new jet was called a kinetic jet. The kinetic jet was determined to result from a non-MHD particle orbit instability that had a distinct threshold. This instability depended on an interaction between two properties of the configuration, namely (i) MHD plasma jets are accelerated from both electrodes into the flux tube (see Fig.5) and (ii) the magnetic field is helical.
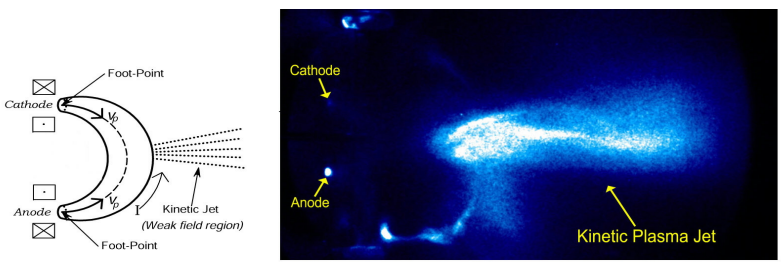

FIGURE 6. Kinetic jet emanating from apex of arched flux tube (from Ref.[8]).

Because jets are accelerated from both electrodes into the flux tube, one of these jets must emanate from the cathode. Furthermore, since the jet consists of quasi-neutral plasma, both electrons and ions are traveling away from the cathode. Thus, the ions in the jet emanating from the cathode are going the wrong way relative to the direction of the current (even though the ions and electrons are both moving away from the cathode, the current which is the difference 
between the ion and electron velocities nevertheless flows from anode to cathode). It will now be shown that if these "wrong-way" ions go sufficiently fast, they are expelled from the flux tube. Since the confinement of ions is weakest at the apex of the arched flux tube where the field is weakest, expelled ions form a jet emanating from the apex; this is the kinetic jet. The threshold for instability can be explained in terms of a Hamiltonian theory whereby at threshold, an "effective potential" valley responsible for confinement of ions to the flux tube transforms to be an "effective potential" hill. Ejection of ions from the flux tube corresponds to the ions falling off the "effective potential" hill.

The threshold can also be seen in an intuitive fashion by consideration of the radial component of the Lorentz force for ions having axial velocity $v_{z}$ in a helical magnetic field. Taking centrifugal force into account, and using $B_{\phi}=\mu_{0} J_{z} r / 2$ for a uniform current density $J_{z}$, the radial component of the Lorentz force equation $m d \mathbf{v} / d t=q \mathbf{v} \times \mathbf{B}$ is

$$
m\left(\ddot{r}-r \dot{\phi}^{2}\right)=q\left(r \dot{\phi} B_{z}-v_{z} \mu_{0} J_{z} r / 2\right) .
$$

In the limiting case $J_{z}=0$ and for $\ddot{r}=0$, Eq.11 reduces to $\dot{\phi}=-q B_{z} / m$, i.e., to Larmor orbital motion. However, if $J_{z} \neq 0$ and a solution with $\ddot{r}=0$ is again sought, Eq. 11 reduces to

$$
\dot{\phi}^{2}+\dot{\phi} \frac{q}{m} B_{z}-\frac{q}{m} v_{z} \mu_{0} J_{z} / 2=0
$$

a quadratic equation in $\dot{\phi}$ with solution

$$
\dot{\phi}=-\frac{q B_{z}}{2 m} \pm \frac{1}{2} \sqrt{\left(\frac{q B_{z}}{m}\right)^{2}+2 \frac{q}{m} v_{z} \mu_{0} J_{z}} .
$$

Thus, if $q v_{z} J_{z}$ is negative (corresponding to wrong-way motion) and if

$$
\left|2 \frac{q}{m} v_{z} \mu_{0} J_{z}\right|>\left(\frac{q B_{z}}{m}\right)^{2}
$$

there is no real solution to the quadratic equation. In such a case it would thus be incorrect to assume $\ddot{r}=0$. The limit of large $\left|q v_{z} \mu_{0} J_{z} / m\right|$ in Eq.11 corresponds to positive $\ddot{r}$ and since $\ddot{r}$ cannot pass through zero if Eq. 14 holds, $\ddot{r}$ must be positive-definite. Thus, if Eq.14 is satisfied, i.e., if the ion velocity is such that

$$
-m v_{z} \mu_{0} J_{z} / q B_{z}^{2}>1 / 2
$$

the ion will be ejected from the flux tube since it will always have $\ddot{r}>0$.

By adjusting the gas puff valves to provide different amounts of gas injection, the jet mass density and hence velocity was varied and the onset of the instability was observed to agree with Eq.15.

Acknowledgments: Supported by USDOE, NSF, and AFOSR.

\section{REFERENCES}

1. S. C. Hsu, and P. M. Bellan, IEEE Transactions on Plasma Science 30, 10-11 (2002), part 1.

2. S. C. Hsu, and P. M. Bellan, Physical Review Letters 90 (2003), Art. No. 215002

3. S. You, G. S. Yun, and P. M. Bellan, Physical Review Letters 95 (2005), Art. No. 045002

4. G. S. Yun, S. You, and P. M. Bellan, Nuclear Fusion 47, 181-188 (2007).

5. D. Kumar, and P. M. Bellan, Physical Review Letters 105 (2009), Art. No. 105003.

6. J. F. Hansen, and P. M. Bellan, Astrophysical Journal 563, L183-L186 (2001), part 2.

7. J. F. Hansen, S. K. P. Tripathi, and P. M. Bellan, Physics of Plasmas 11, 3177-3185 (2004).

8. S. K. P. Tripathi, P. M. Bellan, and G. S. Yun, Physical Review Letters 98 (2007), Art. No. 135002.

9. P. M. Bellan, Spheromaks: a practical application of magnetohydrodynamic dynamos and plasma self-organization, Imperial College Press, London, 2000.

10. S. C. Hsu, and P. M. Bellan, Physics of Plasmas 12 (2005), Art. No. 032103.

11. D. Kumar, and P. M. Bellan, Review of Scientific Instruments 77 (2006), Art. No. 083503.

12. P. M. Bellan, Physics of Plasmas 10, 1999-2008 (2003), part 2.

13. G. S. Yun, Dynamics of Plasma Structures Interacting with External and Self-Generated Magnetic Fields, Ph.D. thesis, California Institute of Technology, Pasadena, CA, 91125, USA (2008).

14. E. V. Stenson, and P. M. Bellan, IEEE Transactions on Plasma Science 36, 1206-1207 (2008), part 1. 